\title{
Upconversion Nanocrystals: Synthesis, Properties, Assembly and Applications
}

\author{
Danny Vennerberg and Zhiqun Lin* \\ Department of Materials Science and Engineering, Iowa State University, Ames, IA 50011, USA
}

\begin{abstract}
Upconversion nanocrystals represent a new class of nonlinear optical materials that can convert low energy radiation into higher energy radiation. Recent advances in synthesis techniques have produced nanocrystals with controllable size and color emission as well as novel surface properties that enable such attributes as water solubility and biological functionality. This in turn has led to an explosion in research activity, primarily targeted towards biological applications. This Review seeks to fill the need for a broad-based introduction to the capabilities of upconversion nanocrystals while emphasizing the properties that could be of use to readers from various disciplines.
\end{abstract}

Keywords: Upconversion Nanocrystals, ${ }^{2}$ Rare-Earth 8 lons, Doped $\mathrm{NaYF}_{4}$ Nanocrystals, Near-Infrared Emission,6 Excited-State 5 Absorption, Luminescence, Nanocrystal Synthesis, Surface Modification, Assembly.

\section{CONTENTS}

1. Introduction

1.1. Upconversion Mechanisms

1.2. Quenching Mechanisms

1.3. Dopant-Host Interaction

2. Synthesis of UCNCs

2.1. Coprecipitation.

2.2. Thermal Decomposition

2.3. Hydro(solvo)thermal Synthesis

2.4. Sol-Gel Synthesis

2.5. Combustion Synthesis

2.6. Flame Synthesis

3. Emission Tuning

3.1. Nanocrystal Size Effects

3.2. Dopant Concentration Effects

3.3. Phase and Morphology Effects

3.4. Excitation Intensity Effects

4. Surface Engineering $\ldots \ldots \ldots \ldots \ldots \ldots \ldots \ldots \ldots \ldots \ldots$

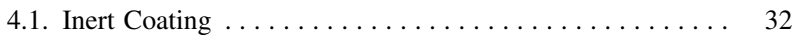

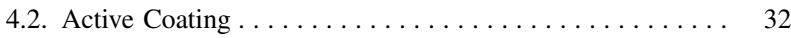

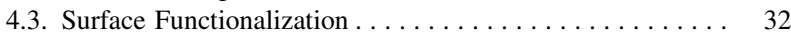

4.4. Plasmonic Enhancement. . . . . . . . . . . . . . . . 34

4.5. Magnetic Functionalization $\ldots \ldots \ldots \ldots \ldots \ldots \ldots \ldots . \ldots \ldots$

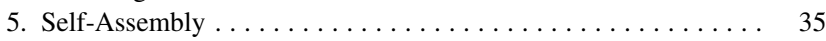

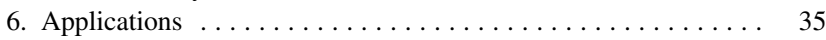

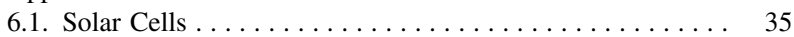

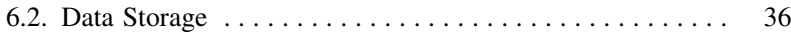

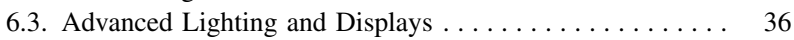

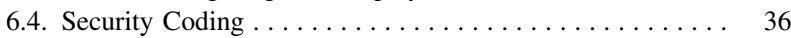

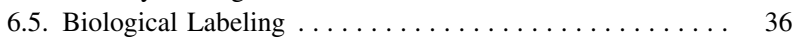

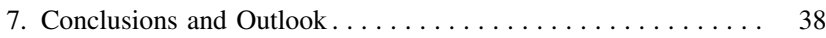

References and Notes $\ldots \ldots \ldots \ldots \ldots \ldots \ldots \ldots \ldots$

*Author to whom correspondence should be addressed.

\section{INTRODUCTION}

The fascinating optical phenomenon of upconversion luminescence has warranted research since the 1960's. Pioneering efforts by Auzel, Ovsyankin, and Feofilov investigated the mechanisms of upconversion in rare earth-doped bulk phosphors. ${ }^{1}$ Novel devices based on near-infrared to visible upconverting materials emerged, including lasers, ${ }^{2}$ infrared quantum counters, ${ }^{3,4}$ temperature sensors, three dimensional displays, ${ }^{5}$ and spectral converters for solar cells. ${ }^{6}$ The study of lanthanide-doped materials laid the foundation for the burgeoning new field of upconversion nanocrystals (UCNCs). While nano-scale upconverting crystals generally lack the efficiency of their bulk counterparts, they possess many intriguing properties such as water solubility via surface engineering and continuous and predictable changes in emission through particle size control. As such, UCNCs have already produced a wide spectrum of applications spanning biology and materials science. For instance, sophisticated biological labels have been developed using core/shell nanoparticles, which enable both fluorescence and magnetic resonance imaging (see Section 6.5). Because UCNCs can be dispersed in commonly used solvents such as water and toluene, they can be readily printed onto a variety of substrates. Recently, this property has been exploited to produce proof-of-concept security marks (see Section 6.4). With the anticipation of continued growth in upconversion research, an overview of UCNC synthesis, assembly, properties, and applications is in order. This Review seeks 
to identify important factors for controlling the properties of UCNCs while providing salient examples of how these unique characteristics can be applied. The first section outlines the basic energy transfer events that occur in UCNCs as well as design considerations important in minimizing quenching events. The second section gives an overview of synthetic routes now available, noting the advantages and disadvantages of each technique. The third section offers strategies for the precise tuning of upconversion emission, and the fourth section provides examples of how surface modification grants UCNCs intriguing properties such as biofunctionality and water solubility. The fifth section touches on the relatively unexplored area of UCNC selfassembly, which has the potential to produce novel optical and electronic devices. The sixth section showcases the wide range of applications that have benefited from UCNCs thus far, with the intent of stimulating further diversity. The final section frames the future outlook of the field by highlighting areas of exceptional promise:

\subsection{Upconversion Mechanisms}

The term upconversion refers to the process of converting low energy incident radiation into higher energy output radiation. Upconversion is a nonlinear optical process that requires two or more metastable energy states to store the energy of absorbed pump photons. The combined energies of pump photons can lead to the emission of a higher energy photon. Much work has been done to study the precise energy transfer processes that occur during an upconversion event. ${ }^{1}$ The complex interactions will not be covered here, but a brief overview of the basic possibilities is given as background for those unfamiliar with nonlinear optics. Four fundamental energy transfer processes are given in Figure 1.

In the first process, excited state absorption (ESA), an ion absorbs a near-infrared pump photon, which excites the ion from the ground state to E2, before absorbing another photon to excite it to E3. The excited ion then returns to the ground state, E1, and gives off radiation with energy (E3-E1).

The second process, cross relaxation (CR), involves two ions, each of which is initially excited by a pump photon to E2. One ion transfers energy to the other, resulting in one ground state ion and one ion excited to E3. The excited ion then releases the energy in the form of a photon. Ions that donate energy are generally referred to as sensitizers, and ions that receive energy and emit radiation are called activators.

The third mechanism, energy transfer upconversion (ETU), involves three ions (two sensitizers and one activator). Two sensitizer ions absorb pump photons to each reach E2. Both ions transfer energy to a third ion, exciting it to E3. The activator then gives off a photon and returns

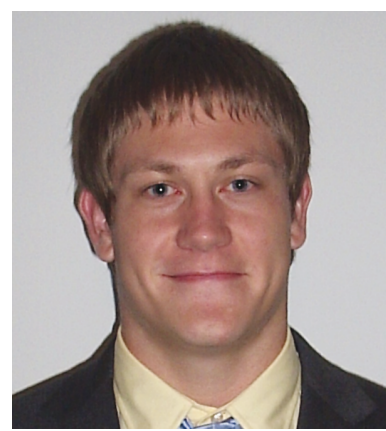

Mr. Danny Vennerberg is concurrently pursuing B.S. and M.S. degrees in the department of Materials Science and Engineering at Iowa State University. In 2008, through the National Science Foundation's REU program, he studied vanadate glass semiconductors and presented the results at the 8th Pacific Rim Conference on Ceramic and Glass Technology. Later, as a research assistant at Ames Laboratory, U.S. Department of Energy, he investigated stress corrosion cracking of steels in ammonia. Since 2009 he has worked in Professor Zhiqun Lin's nanostructured functional materials group. His current research interests include nonlinear optics, functional nanocrystals, photonic materials, and dye-sensitized solar cells.

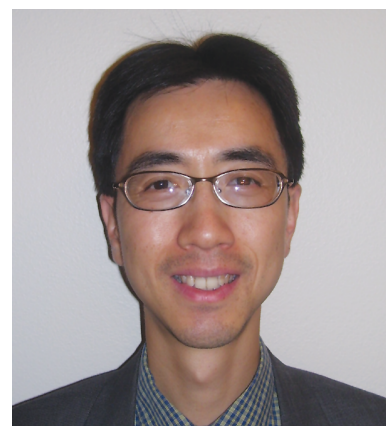

Dr. Zhiqun Lin received the B.S. degree in Chemistry from Xiamen University, Xiamen, China in 1995, the Master degree in Macromolecular Science from Fudan University, Shanghai, China in 1998, and the Ph.D. degree in Polymer Science and Engineering from University of Massachusetts, Amherst in 2002. From November 2002-July 2004 he was a postdoctoral associate at the department of Materials Science and Engineering at the University of Illinois, Urbana-Champaign. He has been an Assistant Professor in the department of Materials Science and Engineering at the Iowa State University since 2004, and was promoted to Associate Professor in 2010. His research interests include solar cells, quantum dots (rods), conjugated polymers, multifunctional materials, polymer-based nanocomposites, block copolymers, polymer blends, nanoscopic structures, hierarchical structure formation and assembly, ferroelectric nanocrystals, multiferroic nanocrystals, negative giant magnetocaloric effect nanoparticles, $\mathrm{TiO}_{2}$ nanoparticles and nanotubes, porous alumina membranes, phase equilibrium and phase separation kinetics, surface and interfacial properties, electrohydrodynamic instabilities, and nanodynamics. He is a recipient of an NSF Career Award and a 3M Non-Tenured Faculty Award, and an invited participant at the National Academy of Engineering's (NAE) 2010 U.S. Frontiers of Engineering Symposium (US FOE). 
(a)

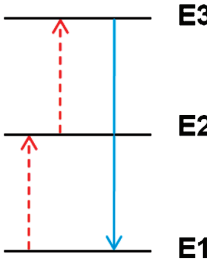

ESA

(c)

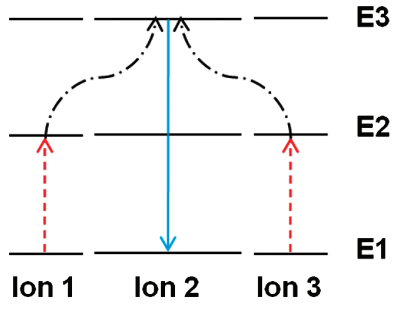

ETU (b)

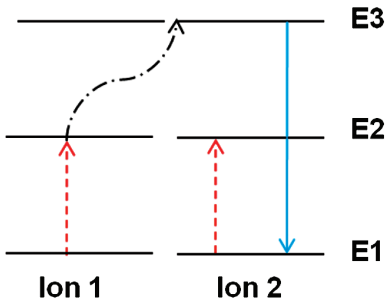

CR

(d)

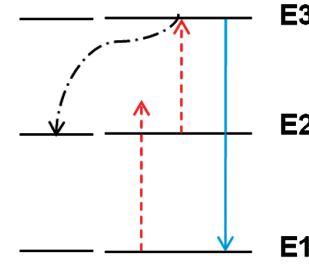

PA
Fig. 1. Four basic energy transfer mechanisms: excited state absorption (a), cross relaxation (b), energy transfer upconversion (c), and photon avalanche (d).

to the ground state. It is worth noting that ETU is the most efficient upconversion mechanism, offering more than an order of magnitude advantage over ESA. ${ }^{1}$ ETU is efficient because it favors resonant absorption, which leads to long excitation lifetimes and increases the probability of upconversion.

The fourth process, photon avalanche, is more complex than the first three and only occurs when the pump photon intensity reaches a critical level. If the incident light is sufficiently intense, the first excited state, E2, of many ions becomes populated via nonresonant ground state absorption. The increase in the number of populated E2 states causes the transparency of higher energy states to decrease, thus leading to a greater number of ESA events. As more ESA occurs, some of the ions excited to E3 transfer energy to surrounding ground state ions, further increasing the population of E2 states and causing an "avalanche" effect of creating more and more excited ions. A detailed account of energy transfer events, including photon avalanche, is given by Auzel $^{1}$ for those seeking a more rigorous treatment.

\subsection{Quenching Mechanisms}

Many deleterious processes can occur which limit the efficiency of upconversion luminescence. Any process that lowers the number of metastable excited states without giving off upconverted radiation is referred to as quenching. Phonons provide a competitive energy transfer route to radiative emission. As such, ions with closely spaced energy levels are susceptible to quenching by multi-phonon transitions to lower energy levels. Multiple phonons are necessary to "bridge" the same energy gap as a single photon, so the probability of phonon quenching decreases exponentially as the energy gap widens. This observation has been coined the "energy gap law" and has been found to follow the general form ${ }^{7}$

$$
A=A_{0} \exp (-\alpha \Delta E)
$$

where $A$ is the multiphonon emission rate, $A_{0}$ and $\alpha$ are host-dependent constants, and $\Delta E$ is the difference of the populated energy level and the next lowest energy level in the lanthanide ion. As a rule of thumb, if the energy gap of an ion is greater than the energy of 6 phonons, radiative emission will dominate, and if the energy gap is smaller than the energy of 6 phonons, nonradiative phonon relaxation will dominate. Another quenching mechanism occurs through radiative emission of low energy photons. If a sublevel exists between the excited state and the ground state, emission may occur in two steps rather than one. Quenching can also occur via $\mathrm{CR}$ to impurities or other lanthanide ions. Therefore, the concentration of dopant ions must be carefully controlled to avoid cross quenching and achieve high efficiency.

\subsection{Dopant-Host Interaction}

Upconverting nanocrystals are generally comprised of an inert host material doped with optically active sensitizer and activator ions. Controlling the mechanisms of energy transfer among these three constituents is of utmost importance when selecting dopant-host pairs. Host materials must have low phonon energies to avoid efficiency losses I via nonradiative transfer and should be chemically stable. To readily incorporate lanthanide dopant ions as substitutional impurities, host materials must be of comparable size to the trivalent rare earths. Fluorides such as $\mathrm{NaYF}_{4}$ and $\mathrm{LaF}_{3}$ meet these criteria and are popular choices as host materials in UCNCs. Oxides such as $\mathrm{ZrO}_{2}, \mathrm{Y}_{2} \mathrm{O}_{3}$, $\mathrm{LuPO}_{4}$ and $\mathrm{YbPO}_{4}$ are also used as host materials because of their chemical stability, though they generally have larger phonon energies than fluorides.

Lanthanide ions have been the primary dopant choice in UCNCs because their ladder-like energy levels allow for metastable storage of pump photon energy. For example, Figure 2 shows the nearly evenly spaced energy levels of $\mathrm{Ho}^{3+}$, which promotes upconversion by the absorption of multiple photons. $\mathrm{Er}^{3+}$ and $\mathrm{Tm}^{3+}$ also have large gaps between energy states that discourage nonradiative energy transfer, making them efficient lanthanide activators. Doping a host material with many activator ions increases UC emission until a critical concentration is reached. Above this concentration, CR quenching among activator ions serves to lower the upconversion emission. To avoid this problem, one or more lanthanide ions are often added in 


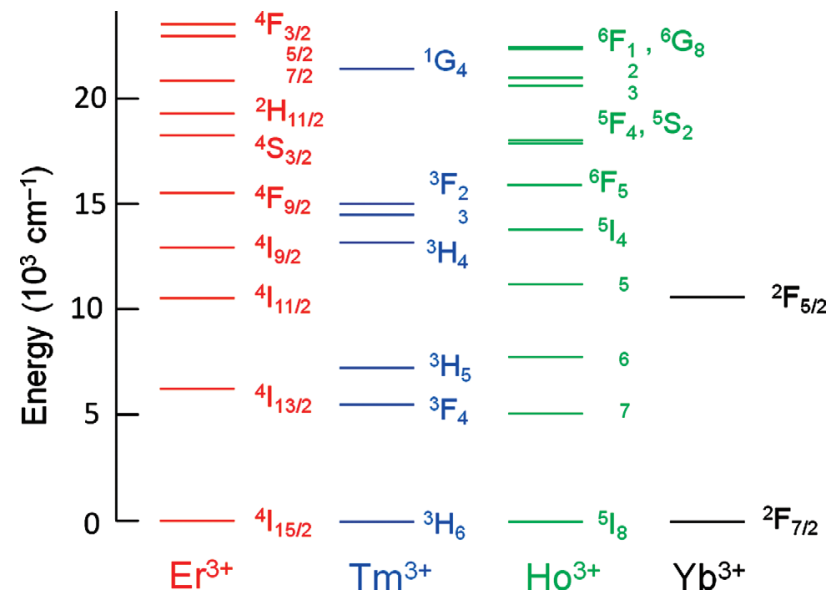

Fig. 2. Energy level diagrams of commonly used lanthanide activator ions and the sensitizer ion, $\mathrm{Yb}$.

large amounts as sensitizers and the concentration of activators is kept low. Sensitizers should have large absorption cross-sections in the infrared and energy levels that are compatible with the activator being used. $\mathrm{Yb}^{3+}$ is $\mathrm{a}$ popular sensitizer because its energy levels are resonant with the $\mathrm{f}-\mathrm{f}$ transitions of many lanthanide activators used $\left(\mathrm{Er}^{3+}, \mathrm{Tm}^{3+}\right.$, and $\left.\mathrm{Ho}^{3+}\right)$, and it has a large near-infrared absorption cross-section. For instance, the $\mathrm{F}^{5 / 2}$ state of $\mathrm{Yb}^{3+}$ nearly overlaps the $\mathrm{I}^{11 / 2}$ state of $\mathrm{Er}^{3+}$, allowing for compatible energy transfer from $\mathrm{Yb}$ to $\mathrm{Er}$, as can be seen from the energy level diagrams in Figure 2.

\section{SYNTHESIS OF UCNCS}

Though bulk upconverting materials have been synthesized for nearly fifty years, only recently have techniques been developed to synthesize lanthanide doped nanocrystals. Several methods for the production of both oxide and fluoride host nanocrystals are now available, and approaches have been developed to address specific needs such as monodispersity and surface functionalization for aqueous solubility. Some of the most useful approaches will be reviewed below. It should be noted that the following processes are not mutually exclusive, and it is often the case that more than one method can be combined to produce UCNCs.

\subsection{Coprecipitation}

Coprecipitation involves the simultaneous precipitation of two substances. With this technique, UCNCs are often precipitated alongside organic surfactants such as oleic acid, which adsorb to the surface of the nanoparticles to control size and prevent agglomeration. Coprecipitation is widely used for the preparation of small $(10-30 \mathrm{~nm})$, nearly monodisperse UCNCs because it does not require precisely controlled reaction times, expensive equipment, or complex procedures. Early attempts by Yi yielded $\alpha$-phase (cubic) $\mathrm{NaYF}_{4}: \mathrm{Yb}$,Er via coprecipitation of $\mathrm{Y}^{3+}, \mathrm{Yb}^{3+}$, and $\mathrm{Er}^{3+}$ with $\mathrm{NaF}$ in the presence of ethylenediaminetetraacetic acid (EDTA). ${ }^{8}$ Subsequent post-precipitation annealing for $5 \mathrm{~h}$ at temperatures between 400 and $600{ }^{\circ} \mathrm{C}$ produced $\beta$-phase (hexagonal) $\mathrm{NaYF}_{4}: \mathrm{Yb}$, Er. The average UCNC size was tuned from 37 to $166 \mathrm{~nm}$ by varying the amount of EDTA. Efforts by Haase's group produced transparent, soluble $\beta$-phase $\mathrm{NaYF}_{4}: \mathrm{Yb}, \mathrm{Er}(\mathrm{Tm}) \mathrm{UCNCs}$ using the coprecipitation method but without the need for a high temperature calcination step. ${ }^{9}$ A more user-friendly technique for synthesis of $\beta$-phase $\mathrm{NaYF}_{4}: \mathrm{Yb}, \operatorname{Er}(\mathrm{Tm})$ UCNCs has been proposed by $\mathrm{Li}$ and Zhang that does not require excess amounts of fluoride reactants at high temperatures, thus eliminating the production of volatile fluorinated species. ${ }^{10}$ Plate-like, sphere-like, and ellipselike nanocrystals have been produced with this method by tuning the oleic acid ligand concentration. Besides controlling the morphology of an UCNC, ligands can also be used to provide sites for functionalization and grant particles water dispersibility. For instance, amphiphilic polyvinylpyrrolidone (PVP) can act as a surfactant in place of the hydrophobic oleic acid ligands used in many other methods. ${ }^{11}$

\subsection{Thermal Decomposition}

Thermal decomposition refers to the decomposition of one substance into two constituents upon heating. Boyer synthesized cubic phase $\mathrm{NaYF}_{4}$ crystals doped with $\mathrm{Yb} / \mathrm{Er}$ or $\mathrm{Yb} / \mathrm{Tm}$ via thermal decomposition of trifluoroacetate precursors mixed with octadecene, oleic acid, and technical grade chemicals. ${ }^{12}$ Impressive monodispersity (the average particle size was $27.6 \pm 1.6 \mathrm{~nm}$ ) and uniformity of shape were achieved. Careful experimental control is necessary during a thermal decomposition reaction as the metal precursors are susceptible to oxidation and toxic byproducts are formed during synthesis. ${ }^{13}$ A recently reported automation process for nanoparticle synthesis optimized the oleic acid/rare earth ratio to produce high efficiency $\mathrm{NaYF}_{4}$ :Yb,Er UCNCs using a thermal decomposition synthesis scheme. ${ }^{14}$ The advent of high-throughput, automated synthesis and characterization systems provides an exciting new paradigm for the production of UCNCs.

\subsection{Hydro(solvo)thermal Synthesis}

Hydro(solvo)thermal methods have been shown to yield UCNCs with well controlled size and shape using inexpensive chemicals. However, they require the use of an expensive autoclave capable of withstanding high temperatures, large pressures, and corrosion. Hydro(solvo)thermal methods work by heating and pressurizing a solvent above its critical point in order to increase solid solubility. Work by Liang produced $\mathrm{NaRF}_{4}$ nanotubes via a hydrothermal method, ${ }^{15}$ where $R$ represents a trivalent rare earth ion. Later, Zeng developed a hydro(solvo)thermal 
technique to produce hexagonal $\mathrm{NaYF}_{4}: \mathrm{Yb}$,Er UCNCs with good dispersibility in ethanol. ${ }^{16}$ The morphology of these UCNCs can tuned using cetyltrimethylammonium bromide (CTAB) and the size can be controlled by the addition of EDTA. An advantage of hydro(solvothermal) synthesis is the ability to vary many experimental parameters such as reaction temperature, reaction time, solvent type, and surfactant type to control the size and morphology of the resulting nanocrystals.

\subsection{Sol-Gel Synthesis}

The sol-gel synthesis method is widely used in the production of oxide and fluoride nanocrystals and thin films. The process begins with a liquid solution of organometallic precursors that form a new sol phase via hydrolysis and polycondensation. The nanocrystals comprising the sol then agglomerate into another gel phase as a large marcromolecular network with the addition of a base. Calcination removes the solvents from the gel and increases crystallinity. Er-doped $\mathrm{TiO}_{2}$ and $\mathrm{BaTiO}_{3}$ nanocrystals were synthesized by Patra using titanium isopropoxide and barium acetate as sol precursors. ${ }^{17} \mathrm{~A}$ similar method has been used by Rosa-Cruz to produce Er-doped $\mathrm{ZrO}_{2} \cdot{ }^{18}$ Guo developed a simple sol-gel technique for synthesizing rare earth-doped $\mathrm{Gd}_{2} \mathrm{O}_{3}$ UCNCs that emit a wide range of visible radiation upon $980 \mathrm{~nm}$ excitation. ${ }^{19}$ Spherical UCNCs of $\mathrm{Gd}_{3} \mathrm{Ga}_{5} \mathrm{O}_{12}$ have also been produced by Daldosso using the Pechini sol-gel method, though a large size distribution was observed. ${ }^{20}$ It should be mentioned that this lack of monodispersity is common to many sol-gel techniques. Recently, Yang reported the synthesis of rare earth fluoride-silica core-shell upconverting nanorods, which utilized a surfactant assisted sol-gel process to coat $\mathrm{NaYF}_{4}: \mathrm{Yb}, \mathrm{Er}$ nanorods with $\mathrm{SiO}_{2} \cdot{ }^{21}$

\subsection{Combustion Synthesis}

Combustion synthesis offers a rapid technique for the creation of UCNPs through a series of controlled explosions. This technique requires reactants which will undergo exothermic reactions when heated by a low temperature source. Vetrone has reported the synthesis of Er-doped $\mathrm{Gd}_{3} \mathrm{Ga}_{5} \mathrm{O}_{12}$ from a solution combustion method that utilizes an exothermic reaction between metal nitrates and an organic fuel such as glycine. ${ }^{22} \mathrm{Y}_{2} \mathrm{O}_{3}, \mathrm{La}_{2} \mathrm{O}_{2} \mathrm{~S}, \mathrm{Gd}_{2} \mathrm{O}_{3}$, and several other oxides have been produced with similar methods. Combustion synthesis is appealing as a scalable production method because it requires only a low energy flame (Bunsen burner) to start the reaction, which is fast and self-sustaining.

\subsection{Flame Synthesis}

Flame synthesis is another high-throughput method of producing UCNCs that is even faster than combustion synthesis. A pioneering effort by Ju's group used gas-phase precursors mixed in stoichiometric amounts to synthesize $\mathrm{Y}_{2} \mathrm{O}_{3}$ :Yb,Er UCNCs with diameters less than $30 \mathrm{~nm} .^{23}$ The flame temperature was found to strongly affect particle size, morphology, and upconversion intensity, providing a simple way to tune these properties. More recently, Ju has shown that the flame synthesis technique can be extended to yield $\mathrm{La}_{2} \mathrm{O}_{3}: \mathrm{Yb}$,Er and $\mathrm{Ga}_{2} \mathrm{O}_{3}: \mathrm{Yb}$,Er UCNCs. ${ }^{24}$ Like combustion synthesis, flame synthesis is a readily scalable, low cost, fast, and efficient process that holds potential for commercial production of UCNCs.

\section{EMISSION TUNING}

The emission spectrum of UCNCs is often a paramount design criterion for the applications mentioned in Section 6 of this Review. For example, solar cells can benefit from the emission of a relatively large range of visible radiation, whereas biological labels require narrow, well-defined emission peaks. The ability to easily tune the color and intensity of emitted radiation will make UCNCs convenient for use in a large range of applications.

\subsection{Nanocrystal Size Effects}

The size of UCNCs has been shown to affect emission spectra by (i) determining the number and proximity of surface defects, (ii) restricting phonon relaxation, (iii) influencing the free energy of phases, and (iv) affecting particle geometry. Small particles have large surface area to volume ratios which cause a greater number of nonradiative transfer events with quenching sites on the surface, and hence, lower emission intensities than bulk materials. ${ }^{25}$ Small particles also restrict phonon relaxation via the photon confinement effect, which results in the inaccessiblity of low phonon energy pathways. ${ }^{26}$ This in turn leads to a reduction in energy transfer events among sensitizers and activators, which often require a low energy phonon to deal with a slight excess or deficiency of energy between the two energy levels. Mai has shown that $\alpha$ and $\beta-\mathrm{NaYF}_{4}: \mathrm{Yb}, \mathrm{Er}$ UCNCs exhibit increasing $\mathrm{f}_{\mathrm{g} / \mathrm{r}}$ (ratio of green to red emitted intensities) as particle size increases up to a limiting point at which the $\mathrm{f}_{\mathrm{g} / \mathrm{r}}$ of the particle matches that of the bulk $\left(\approx 70 \mathrm{~nm}\right.$ for $\left.\beta-\mathrm{NaYF}_{4}: \mathrm{Yb}, \mathrm{Er}\right){ }^{25}$ Shan and Ju found that particle size and emission intensity followed a linear relationship for $\beta-\mathrm{NaYF}_{4}: \mathrm{Yb}$,Er synthesized with OA-TOP ligands. ${ }^{27}$ It should be emphasized, however, that particle shape also plays a role in emission intensity, so care must be taken when comparing intensities even for the same phase and size.

\subsection{Dopant Concentration Effects}

Many studies have shown that activator and sensitizer dopant concentrations dramatically affect upconversion luminescence. ${ }^{28-35}$ Wang and Liu reported a general 
approach to multicolor fine tuning of UCNCs by doping $\mathrm{NaYF}_{4}$ with varying amounts of $\mathrm{Ln}^{3+}$ ions. ${ }^{31}$ Using $\mathrm{Yb}$ and $\mathrm{Er}$ as binary dopants, the UCNC emission color could be tuned from yellow to red, and using three dopants $(\mathrm{Yb}, \mathrm{Er}$, and $\mathrm{Tm})$, emission was tuned from blue to white as shown in Figure 3. Li's group used a similar approach to tune the emission color of $\beta-\mathrm{NaYF}_{4}$ nanorods from the UV to IR under excitation from a single wavelength (980 $\mathrm{nm}$ ) by tri-doping with trivalent $\mathrm{Gd}, \mathrm{Er}, \mathrm{Yb}$, and $\mathrm{Tm}$ ions. ${ }^{32}$ Recently, a doping technique has been developed to simultaneously control the phase and size of UCNCs. ${ }^{33}$ By adding precise amounts of $\mathrm{Yb}, \mathrm{Er}, \mathrm{Gd}$, and $\mathrm{Tm}, \alpha$ and $\beta-\mathrm{NaYF}_{4} \mathrm{UCNCs}$ with emissions ranging from green to blue and sizes down to $10 \mathrm{~nm}$ were produced. It was found that $\mathrm{Gd}^{3+}$ has a large impact on the phase and size of $\mathrm{NaYF}_{4}$, while secondary dopant ions such as $\mathrm{Tm}^{3+}$ have a large impact on emission color without changing the size or phase.

\subsection{Phase and Morphology Effects}

The phase and morphology of UCNCs significantly influences their emission spectra. For instance, bulk $\beta-\mathrm{NaYF}_{4}$ has about an order of magnitude higher emission intensity than bulk $\alpha-\mathrm{NaYF}_{4} \cdot{ }^{36}$ Comparisons of UCNCs with the same size but different morphologies have given conflicting results as reported in the literature. For example, opposite observations of the $f_{g / r}$ dependence on the size of $\mathrm{NaYF}_{4} \mathrm{UCNCs}_{\text {have been reported. }}{ }^{25,37}$ A recent study by Shan and Ju addressed this issue by comparing the UC emission from differently shaped UCNCs on the basis of surface area to volume ratios. ${ }^{38}$ It was concluded that the shape effect is independent of size in that particle shape controls lattice energies, and thus mulitphonon relaxation processes, regardless of size. However, size was also found to be important for the previously mentioned reasons of surface defects and restricted phonon relaxation. Ma et al. have recently demonstrated that the morphology of $\mathrm{NaYF}_{4}: \mathrm{Yb}$,Er plays a large role in upconversion emission, ${ }^{39}$ showing that luminescence intensity was greatest from nanowires, less for rods, and least for prisms of the same size.

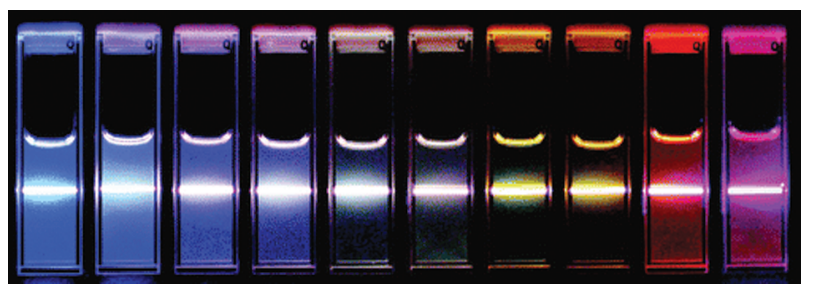

Fig. 3. Upconversion spectra of $\mathrm{NaYF}_{4}: \mathrm{Yb}, \mathrm{Er}(\mathrm{Tm})$ nanocrystals in ethanol obtained after excitation with a $600 \mathrm{~mW} 980 \mathrm{~nm}$ diode laser. The different colors result from variations in the amounts of Tm and Er in the UCNCs. Reproduced with permission from [31], F. Wang and X. G. Liu, J. Am. Chem. Soc. 130, 5642 (2008). (C) 2008, American Chemical Society.

\subsection{Excitation Intensity Effects}

Since upconversion is a nonlinear process, the incident radiation power used to excite an UCNC has a major impact on its emission profile. Pollnau et al. have derived a model for the simplest upconversion excitation schemes involving the absorption of $n$ IR photons per visible photon emitted when only one lanthanide ion is present, in other words, for the case of no sensitizer ions and only activators. ${ }^{40}$ The infrared excitation power, $I_{\mathrm{IR}}$, for a given state is related to its emission intensity, $I$, by the following relation:

$$
I \propto I_{\mathrm{IR}}^{n}
$$

By plotting emission intensity versus excitation power on a $\log -\log$ scale, $n$ can be obtained from the slope. This enables the deduction of the physical nature of UC processes from experimental data since $n$ depends on competition between upconversion processes and linear decay. Pollnau's model predicts that the highest excited state (requiring $n$ excitation photons) will approach $\mathrm{P}^{1}$ as power is increased and lower excited states (requiring $m$ photons where $m<n$ ) will approach $P^{m / n}$ at the high power limit. Suyver has put forth another model to describe the more common case of having sensitizer and activator ions present together. This model shows that when sensitizer ions are present, the high power limit for all states ( $m$ through $n$ ) is 1, resulting in a linear dependence of emission intensity on incident power. Figure 4

(a)
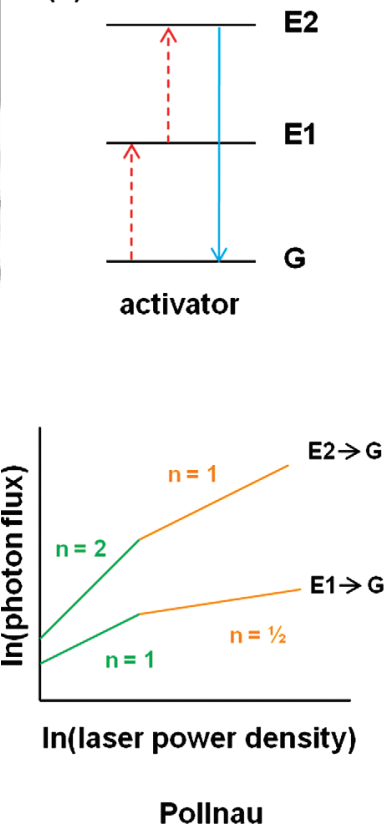

Fig. 4. Schematic depiction of the upconversion intensity dependence on excitation power as predicted by the Pollnau model (a) and the Suyver model (b), where green represents the low power limit and orange the high power limit. The transitions represented on the graphs are labeled to the right of their respective lines and correspond to the energy level diagrams above. 
schematically depicts both the Pollnau and Suyver models for the simplified case of an ion having only three energy levels. $n$ and $m$ represent the number of energy transfer steps needed to reach a given state (the number of rungs climbed on the energy ladder). The relative intensities of different colors emitted will also vary with power up to a point. Suyver and coworkers have shown that above a certain saturation power $\left(\approx 40 \mathrm{~W} / \mathrm{cm}^{2}\right.$ for $\mathrm{NaYF}_{4}: 2 \% \mathrm{Er}$, $18 \% \mathrm{Yb})$, the relative color intensities becomes constant. ${ }^{41}$ This occurs because once the fraction of excited activators approaches unity, upconversion processes become the main relaxation mechanism, replacing other nonradiative depopulation mechanisms. ${ }^{42}$

\section{SURFACE ENGINEERING}

One of the greatest advantages of working with nanocrystalline phosphors is the ability to tailor their surfaces. Many interesting properties can arise from the addition of ligands, coatings, and metals to the surface of UCNCs including aqueous solubility, biological functionality, and plasmonic enhancement.

\subsection{Inert Coating}

Small particle sizes introduce a greater density of defects per dopant ion, which leads to quenching of UC luminescence. For instance, Zhao et al. has reported that adsorbed citrate ligands and hydroxyl groups lead to the quenching of green emission in $\mathrm{NaYF}_{4}: \mathrm{Yb}, \mathrm{Er} \mathrm{UCNCs}{ }^{43}$ To overcome this, many groups have reported the use of an inert shell surrounding the luminescenct La-doped core. Guo et al. demonstrated that the upconversion fluorescence of $\mathrm{NaYF}_{4}: \mathrm{Yb}$,Er UCNCs could be enhanced by the growth of a $\mathrm{NaGdF}_{4}$ shell. ${ }^{44}$ Chow's group reported a 15 fold increase in emission intensity over $\mathrm{NaYF}_{4}: \mathrm{Yb}, \mathrm{Er}$ using a $\mathrm{NaYF}_{4}: \mathrm{Yb}, \mathrm{Er} / \mathrm{NaYF}_{4}$ core/shell structure. ${ }^{45}$ They found that a $3 \mathrm{~nm}$ shell of $\mathrm{NaYF}_{4}$ provided the same luminescence boost as thicker shells, and it was shown that an amorphous shell could be grown on top of the $\mathrm{NaYF}_{4}$ layer with no loss in intensity. Chow also reported that a core/shell structure of $\mathrm{NaYF}_{4}: \mathrm{Yb}, \mathrm{Tm} / \mathrm{NaYF}_{4}$ produced luminescence with 29 times the intensity of $\mathrm{NaYF}_{4}: \mathrm{Yb}, \mathrm{Tm}^{46}{ }^{4}$ In addition to increasing emission efficiency, inert shells can also be used to tune the optical properties of UCNCs. Pichaandi et al. showed that amorphous silica surrounding a $\mathrm{LaF}_{3}: \mathrm{Yb}, \mathrm{Ho} \mathrm{UCNC}$ leads to red-shifting of the emission, ${ }^{47}$ and they were able to tune the ratio of red to green emission from $1: 2.3$ to $23: 1$ by simply changing the fraction of crystallinity of the silicon surrounding the UCNPS.

\subsection{Active Coating}

Capobianco's group recently reported the development of active-core/active-shell UCNCs. ${ }^{48} \mathrm{NaGdF}_{4}: \mathrm{Er}, \mathrm{Yb} /$
$\mathrm{NaGd}_{4}: \mathrm{Yb}$ active core/active shell nanoparticles were found to have greatly improved luminescence intensity over $\mathrm{NaGdF}_{4}: \mathrm{Er}, \mathrm{Yb} / \mathrm{NaGdF}_{4}$ active core/inert shell particles. This intensity boost was attributed to the fact that the $\mathrm{Yb}^{3+}$ ions in the active shell were spatially separated from the $\mathrm{Yb}^{3+}$ ions in the active core, thus reducing the number of cross quenching events. This in turn led to efficient sensitization of the $\mathrm{Er}^{3+}$ activator ions in the core shell. The proposed charge transfer mechanism of the activecore/active-shell UCNCs is shown in Figure 5.

\subsection{Surface Functionalization}

Many applications of UCNPs require water solubility and biocompatibility. Several techniques have been investigated to render functionality to UCNPs, with special attention given to $\beta-\mathrm{NaYF}_{4}$ based nanoparticles, as they produce the greatest luminescence intensities known. ${ }^{36}$ Aqueous solubility and functionality have been granted to hydrophobic UCNCs via a variety of surface modifications that can be broadly divided into two main categories: silanization or coating with silica to covalently bond ligands to UCNCs and adsorption of organic ligands to produce a hydrophilic shell via hydrophobic or electrostatic forces.

\subsubsection{Surface Silanization}

Silanization is an attractive functionalization route because silica is biologically and chemically stable and can be easily functionalized by co-hydrolysis of silanes, allowing for conjugation with biological molecules. ${ }^{49}$ Surface silanization can be done on hydrophobic or hydrophilic particles, and this flexibility has made it the most popular method of functionalizing nanocrystals. ${ }^{50}$ UCNCs having hydrophobic ligands such as oleic acid can be coated with silica using a reverse microemulsion method. ${ }^{51}$ This method has the advantage of being simple while allowing the silica shell thickness to be finely tuned, though particle aggregation has been observed when using this technique. ${ }^{52}$ UCNCs having hydrophilic ligands can be coated with silica using the well established Stöber method. Li et al. demonstrated the versatility of silica coatings by encapsulating quantum dots and fluorescent dyes into core/shell $\mathrm{NaYF}_{4}: \mathrm{Yb}, \mathrm{Er}(\mathrm{Tm})$ nanoparticles to yield proof-of-concept FRET sensors. ${ }^{51}$

\subsubsection{Organic Ligand Adsorption}

Several strategies have been developed to introduce a protective outer shell of ligands onto UCNCs, and a few of the most successful will be covered here. Amphiphilic polymers can impart water solubility to UCNCs that have adsorbed hydrophobic ligands through a process of ligand addition. The hydrophobic ends of amphiphilic polymers will intercalate with the hydrophobic ligands 


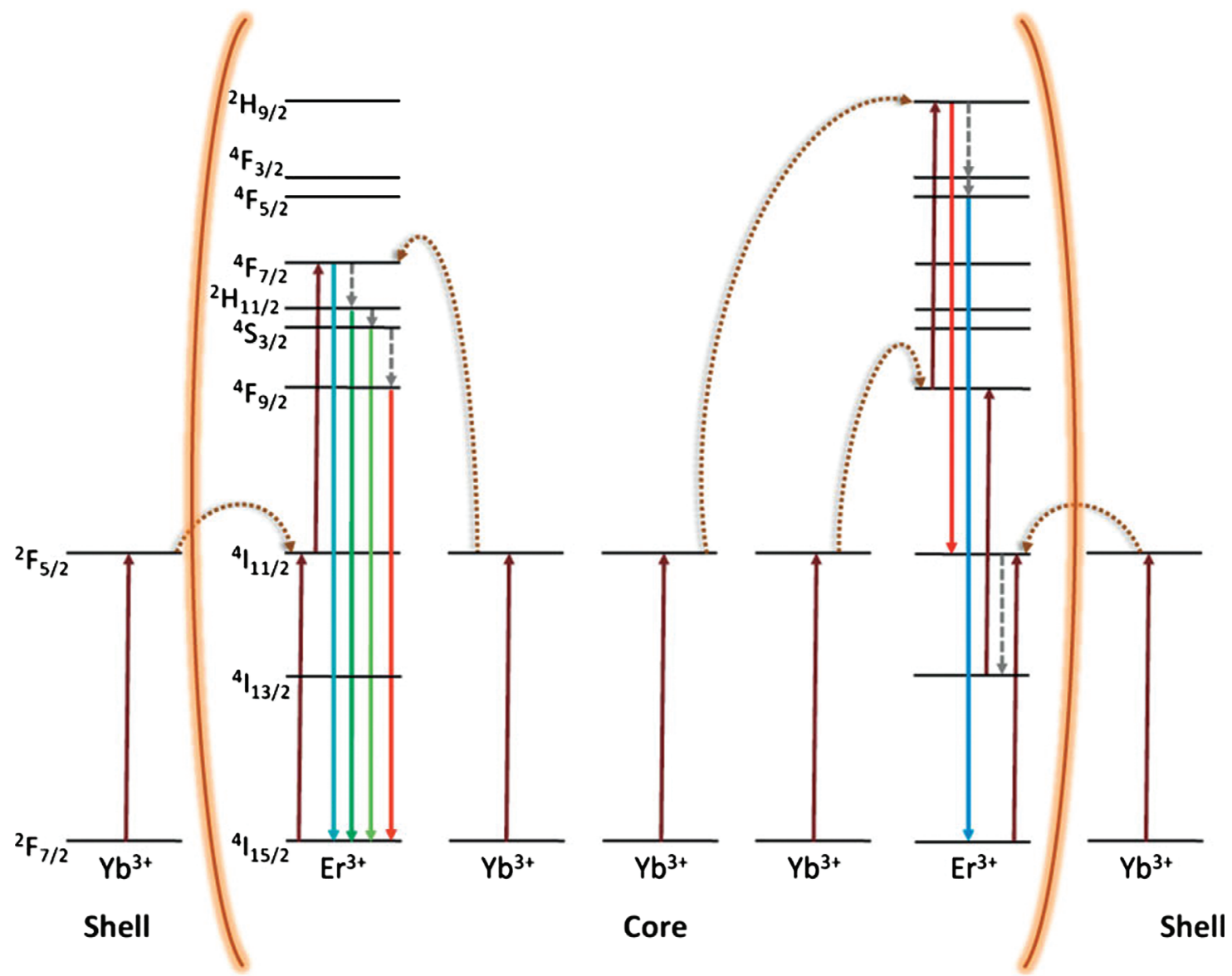

Fig. 5. Schematic energy transfer mechanism of the active core/active shell structure reported in Ref. [48]. Upward pointing arrows represent energy absorption, downward arrows correspond to photon emission, dotted arrows indicate nonradiative multiphonon relaxation, and curved arrows illustrate energy transfer. Reproduced with permission from [48], F. Vetrone et al., Adv. Funct. Mater. 19, 2924 (2009). () 2009, Wiley-VCH.

attached to the UCNCs while the hydrophilic ends of the amphiphilic polymer will spread out into the water. For example, Yi and Chow rendered core/shell $\mathrm{NaYF}_{4}: \mathrm{Yb}, \mathrm{Er}(\mathrm{Tm}) / \mathrm{NaYF}_{4} \mathrm{UCNCs}$ hydrophilic by adding the amphiphilic polymer poly(acrylic acid) (PAA). ${ }^{46} \mathrm{Sim}-$ ilarly, Song's group has shown that thyoglycolic acid (TGA) can be used to make lanthanide doped $\mathrm{NaYF}_{4}$ nanocrystals water-soluble. ${ }^{53}$

Instead of attaching to preexisting ligands, new ligands can also replace old ones. Ligand exchange is a widely used strategy to make nanoparticles water soluble by substituting hydrophilic ligands for hydrophobic ones. De Palma et al. have shown that ferrite nanoparticles can be functionalized by a variety of silanes via ligand exchange. ${ }^{54}$ Van Veggel's group has shown that poly(ethylene glycol)-phosphate (PEG-phosphate) ligands can be used to make a variety of UCNCs hydrophilic, including various oxides, $\mathrm{LaF}_{3}$, and $\mathrm{NaYF}_{4} \cdot{ }^{55}$ Ligand exchange of oleic acid with commercially available PVP has recently been conducted in $\beta-\mathrm{NaYF}_{4} \mathrm{UCNCs}$ by van Veggel, ${ }^{49}$ yielding water-soluble particles that are ideal for in vivo biological studies. Kumar et al. has produced carboxyl-terminated UCNCs via ligand exchange of oleic acid with 3-mercaptopropionic acid, which can easily be further functionalized with biorecognizable molecules. ${ }^{56}$

Another surface modification strategy is the oxidation of ligands attached to UCNCs. This technique requires a hydrophobic ligand with a $\mathrm{C}-\mathrm{C}$ double bond, such as oleic acid, that can react with the Lemieux-von Rudloff reagent, permanganate, or other oxidizing agents. $\mathrm{Li}$ et al. used the Lemieux-von Rudloff reagent to oxidize the $\mathrm{C}=\mathrm{C}$ bonds of oleic acid-capped $\mathrm{NaYF}_{4}: \mathrm{Yb}$,Er UCNCs, yielding carboxylic acid groups at the surface. ${ }^{57}$ Yan et al. have introduced a new oxidation method that uses ozone molecules as oxidizing agents. ${ }^{58}$ This method can produce carboxyl or aldehyde functionality, allowing for easy conjugation with a variety of organic molecules.

Instead of chemically altering the ends of preexisting ligands, a layer-by-layer (LBL) assembly method utilizes electrostatic attraction to build up a hydrophilic shell. Following the general approach of Decher, ${ }^{59}$ Wang et al. have demonstrated LBL assembly using negatively charged poly(sodium 4-styrenesulfonate) (PSS) and positively charged poly(allylamine hydrochloride) (PAH) ${ }^{60}$ resulting in uniform coating and precise control of the shell thickness. 


\subsection{Plasmonic Enhancement}

The phenomenon of enhanced emission intensity from fluorescent materials placed near metallic nanoparticles has been well-established. ${ }^{61,62}$ The enhancement is caused by electric fields created by surface plasmon resonance, which involves the interaction of light with a metal to cause cooperative electron-cloud oscillations on the metal surface. While plasmonic effects can enhance excitation and emission, they can also cause quenching via energy transfer to the metal particles. ${ }^{63}$ Zhang et al. demonstrated that growing gold nanoparticles on the surface of $\mathrm{NaYF}_{4}: \mathrm{Yb}, \mathrm{Tm}$ nanocrystals can more than double their upconversion intensity, while the growth of a continuous shell was found to quench upconversion luminescence due to scattering of incident excitation radiation. ${ }^{64}$ Table I shows a schematic depiction of the growth of $\mathrm{Au}$ seeds on an UCNC (purple sphere). Schientenger et al. also established plasmon emission enhancement by gold nanoparticles near $\mathrm{NaYF}_{4}: \mathrm{Yb}, \mathrm{Er}$ nanocrystals. ${ }^{63}$ Using a modified AFM to control the spacing of gold nanospheres and UCNCs, emission was monitored as a function of distance between the two particles, and enhancement factors as high as 3.8 were observed. In addition to gold, silver has been pursued as a plasmonic enhancer for UCNCs. A $\mathrm{Ag} / \mathrm{SiO}_{2} / \mathrm{Y}_{2} \mathrm{O}_{3}$ :Er core/shell/shell structure has recently been reported by Zhang et al. ${ }^{65}$ Through tuning of the silica spacer layer (the blue shell in Table I), emission intensities 4 times greater than pure $\mathrm{Y}_{2} \mathrm{O}_{3}$ :Er were achieved. The study of plasmon-enhanced upconversion is still in its infancy, and much research is needed in this area to realize the full potential of plasmonic UCNCs.

\subsection{Magnetic Functionalization}

Like plasmonic enhancement, magnetic functionalization of UCNCs has only recently been explored. By doping host nanoparticles with lanthanide ions having a large number of unpaired electrons, such as $\mathrm{Gd}^{3+}$, UCNCs can posses the dual functionalites of upconversion luminescence and paramagnetism. Such properties make these hybrid UCNCs very attractive for use as bioimaging agents (see Section 6.5). Li et al. reported the synthesis of $\mathrm{NaYF}_{4}: \mathrm{Yb}, \mathrm{Er}(\mathrm{Tm}) / \mathrm{Gd}^{3+}$-Si-DTTA core/shell UCNCs that exhibited dual paramagnetic and upconversion properties. ${ }^{66} \mathrm{Si}$-DTTA was added as an MRI contrast

Table I. Various core/shell strategies used to modify UCNC properties. Representative examples of each approach are given alongside their schematic depiction.

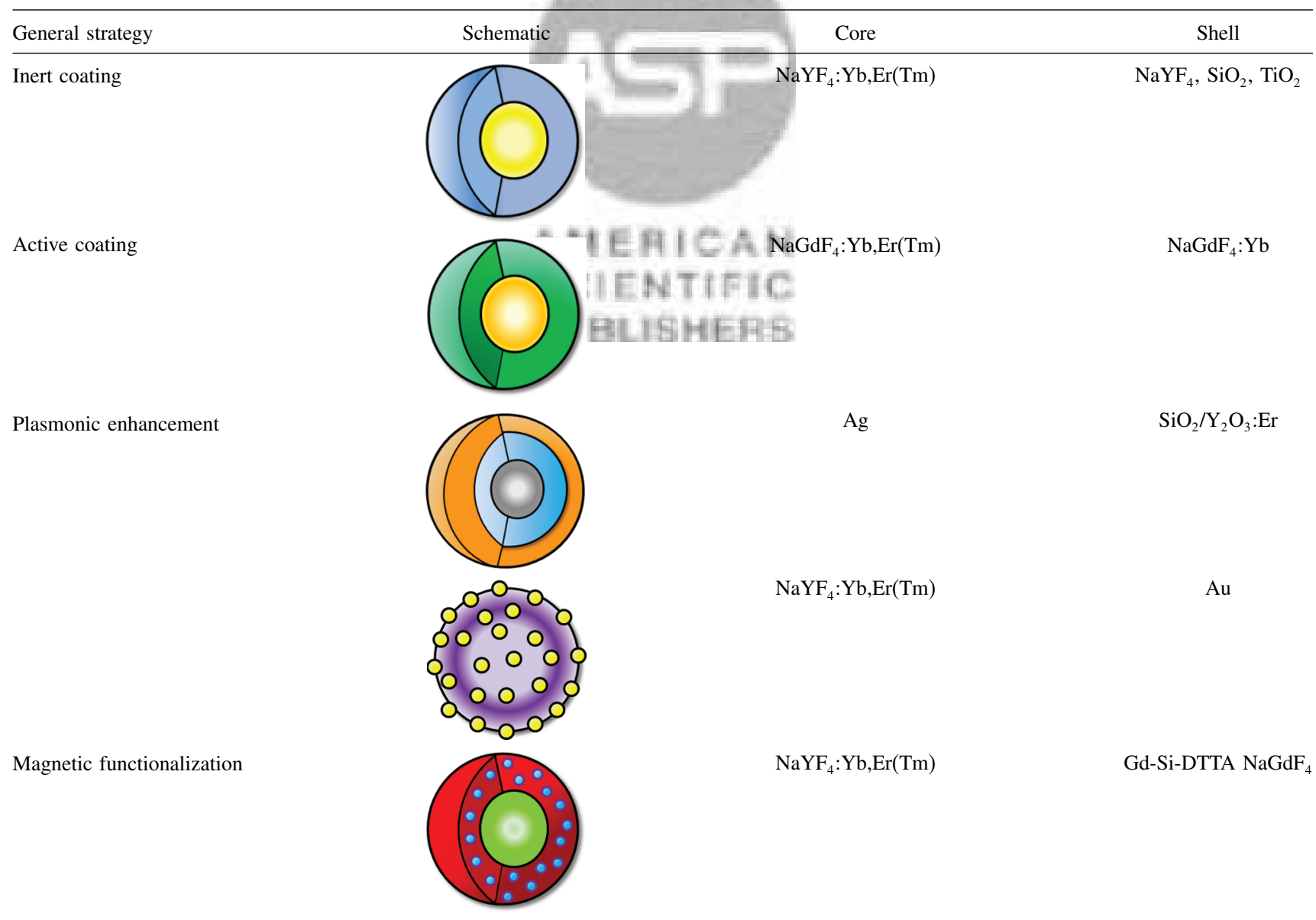


agent to prove the concept of medical imaging. Table I shows the layered structure that was produced. A thin silica shell (red) was deposited on the UCNC core using tetraethyl orthosilicate (TEOS) in a microemulsion technique. Subsequently, Si-DTTA molecules (blue dots) covalently bonded with the TEOS throughout the silica shell during hydrolysis in a basic solution. A slight decrease in luminescence intensity occurred as a result of the silica shell, but sharp emission peaks and good photostability were observed. Guo has very recently shown that paramagnetism can be granted to UCNCs using a seed-mediated epitaxial growth method. ${ }^{44}$ Core/shell $\mathrm{NaYF}_{4}: \mathrm{Yb}, \mathrm{Er} / \mathrm{NaGdF}_{4}$ nanoparticles were synthesized by heating $\mathrm{NaYF}_{4}: \mathrm{Yb}$, Er nanoparticles in a solution of $\mathrm{GdCl}_{3}$, $\mathrm{NaOH}, \mathrm{NH}_{4} \mathrm{~F}$, oleic acid, and octadecene. The $\mathrm{NaGdF}_{4}$ was shown to protect the core from surface quenching and improve upconversion luminescence while also displaying MR relaxivity.

\section{SELF-ASSEMBLY}

Self-assembly is a powerful and widely used tool to produce nano-scale structures. Current work on the self-assembly of UCNCs has yielded intriguing structures that can be used to tune upconversion emission. Zhao's group used the MIMIC method to create upconverting inverse opal structures via the self-assembly of $\mathrm{NaYF}_{4}: \mathrm{Yb}$,Er nanocrystals inside the voids of a PS template. ${ }^{67}$ Hierarchically- ordered, three-dimensional photonic crystal arrays were produced, and by varying the photonic crystal diameter, the photonic stop band could be controlled (Fig. 6). UC emission where the luminescence wavelengths overlapped the stop band was shown to be depressed, and correspondingly, the photoluminescent lifetime of $\mathrm{Er}^{3+}$ ions increased $37 \%$. Ma et al. demonstrated the self-assembly of $1-\mathrm{D} \beta-\mathrm{NaYF}_{4}$ nanowire bundles via a hydrothermal method using SDBS, which displayed a much higher emission intensity than microrods of the same composition. ${ }^{39}$ Chen et al. have recently reported the synthesis of $\mathrm{NaYF}_{4}$ nanocrystals via a rapid, microwaveassisted reaction scheme conducted in fluorine-containing ionic liquid. ${ }^{68}$ Self-assembly of these UCNCs in the ionic liquid resulted in the formation of spherical nanoclusters with controllable size and intense upconversion emission. Continuous exploration of self-assembly promises simple routes to UCNC-based optical and electronic devices.

\section{APPLICATIONS}

Upconverting materials have enjoyed practical uses throughout their development, starting with quantum counters developed in the 1960 's. ${ }^{4}$ A recent surge in UCNC research for biomedical uses has produced a wealth of literature. Therefore, it is not feasible to cover the entire

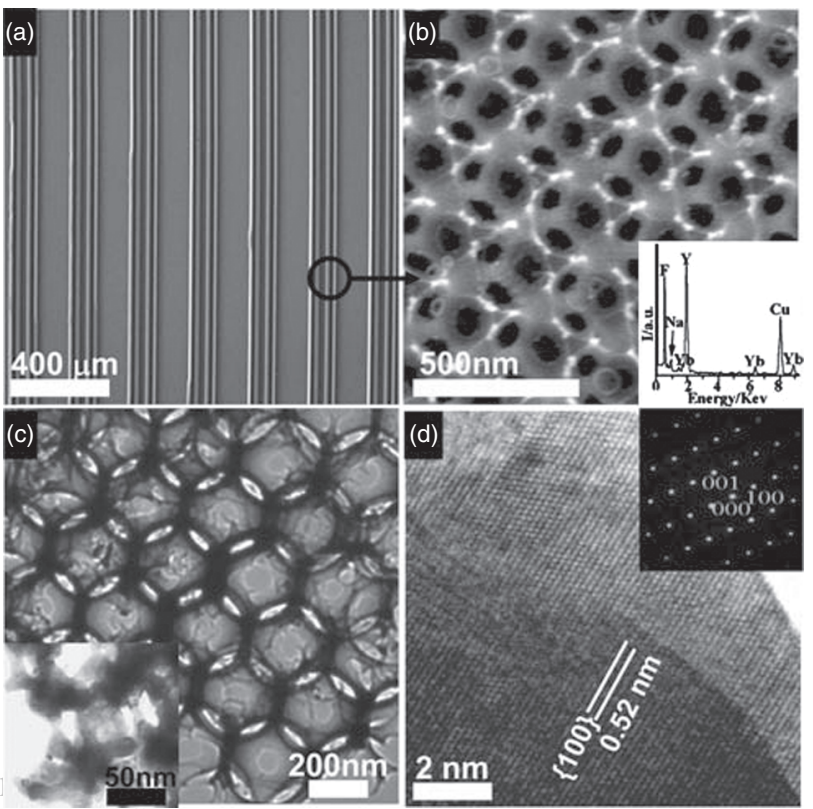

Fig. 6. Self-assembled $\mathrm{NaYF}_{4}: \mathrm{Yb}$,Er photonic crystals reported in Ref. [67]. The hierarchical structure of $\beta-\mathrm{NaYF}_{4}: \mathrm{Yb}, \mathrm{Er}$ is visible from the $\operatorname{SEM}(\mathrm{a}, \mathrm{b})$ and TEM (c) images. The inset (b) is a sample EDAX pattern, and the inset (c) shows a high magnification TEM image of the cellular structure. HRTEM of the $\alpha-\mathrm{NaYF}_{4}: \mathrm{Yb}, \mathrm{Er}$ inverse opal structure is given in (d), and the inset shows an SAED pattern taken on the nanocrystal array. Reproduced with permission from [67], F. Zhang et al., J. Mater. Chem. 20, 3895 (2010). (C) 2010, Royal Society of Chemistry.

spectrum of applications here, though the diversity of suitable functions for UCNCs should serve as a testament to their usefulness. Samples of emerging and thriving research areas are given below to provide both an overview of past work and to promote future efforts.

\subsection{Solar Cells}

Solar cells have the potential to benefit greatly from the addition of upconversion because the band gap of a solar cell inherently limits the amount of solar spectrum that can be collected. A model by Shalav et al. predicts that the maximum efficiency limit of an ideal semiconductor under AM $1.5 \mathrm{G}$ illumination is $31 \%$ for a band gap of $1.35 \mathrm{eV} .{ }^{69}$ Upconversion has the potential to increase that figure by limiting sub-band gap losses, especially for large band gap solar cells. Trupke et al. have proposed a theoretical solar cell configuration that uses upconversion processes to achieve efficiencies of $63.2 \%$ and $47.6 \%$ for nonconcentrated and concentrated sunlight, respectively. ${ }^{70}$ Lanthanide-doped upconversion bulk phosphors have been studied for use as spectral converters since the 1960's; ${ }^{69}$ the reader is referred to an excellent review of the utilization of lanthanide-doped materials in solar cells. ${ }^{6}$ However, the use of UCNCs as spectral converters is limited. The unique geometries made possible by nanocrystalline 
upconverters (such as the self-assembled photonic structure mentioned in Section 5) provide new routes towards the development of efficient solar cells.

\subsection{Data Storage}

As the pursuit for ever increasing data storage densities continues, new approaches will be needed to be developed to enable further improvements. UCNCs have the potential to act as optical storage media, and, when assembled into two or three dimensional periodic arrays, they offer high storage density. Zhang et al. have very recently provided proof-of-concept 2D rewritable storage devices. ${ }^{71} \mathrm{~A}$ composite film consisting of alternating layers of organic and inorganic materials was grown on a silicon substrate. The organic layer contained a photoluminescent diaryl-ethene derivative, 1-\{4-(5-methoxy-2-(2-pyridyl)thiazolyl)\}-2-\{3(2-methylbenzo[b]-thiophenyl)\}hexafluorocyclopentene, which can be ring-opened or ring-closed upon excitation with visible or UV light, respectively. The inorganic layer consisted of $\beta-\mathrm{NaYF}_{4}: \mathrm{Yb}, \mathrm{Er}$ nanocrystals fabricated into a film via spin-coating, the Langmuir-Blodgett method, or evaporation-induced self-assembly. The storage device allowed data to be "erased" using UV radiation, "written" using visible radiation, and "read" using near-IR radiation (since one of the isomers quenched UC emission while the other one did not). By using UCNCs requiring twophoton processes and low energy excitation, the problem of destructive readout (from photoisomerization) was circumvented. Further work regarding the self-assembly and patterning of 2D and 3D UCNC templates will enable more complex storage devices to be fabricated and allow for higher data densities.

\subsection{Advanced Lighting and Displays}

The ability to disperse UCNCs in a transparent polymer matrix has led to the development of novel optical displays. $\mathrm{NaYF}_{4}$-PMMA composites with the capability to emit white, green, and blue light have been fabricated. ${ }^{72,73}$ Following ground-breaking work by Downing, ${ }^{5}$ Wang et al. showed that UCNCs can be used for 3D displays. ${ }^{33}$ The image was created with a $\mathrm{NaYF}_{4}$-PDMS composite and a computer directed $980 \mathrm{~nm}$ laser (Fig. 7). The relatively modest cost of continuous wave infrared lasers makes the large scale use of these displays feasible, and additional work can be done to find UCNCs with low saturation powers (see Section 3.4) in order to reduce the IR laser power necessary to achieve stable color output.

\subsection{Security Coding}

The unique photoluminescent properties of UCNCs also make them potentially useful in anti-counterfeiting marks. Many UCNCs are dispersible in common, inexpensive solvents, making them easy to print or spin coat onto a
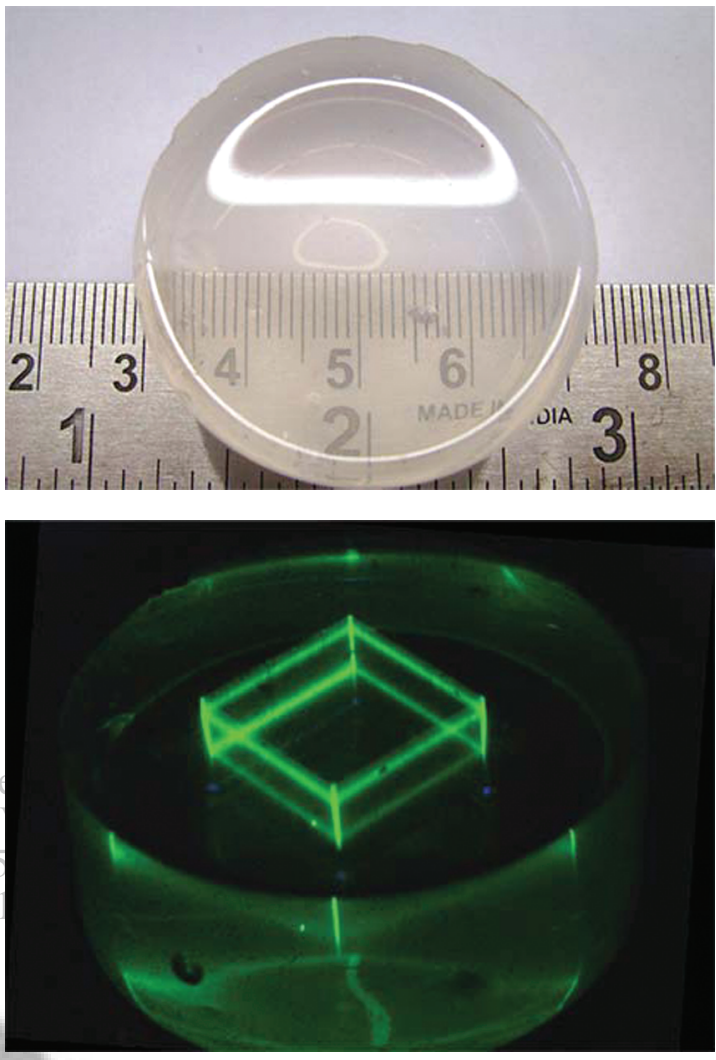

Fig. 7. Composite display material composed of $0.1 \mathrm{wt} \%$ $\mathrm{NaYF}_{4}: \mathrm{Yb}, \mathrm{Er}, \mathrm{Gd}$ UCNCs dispersed in PDMS. The dimensions and transparency of the disk are demonstrated in the top image, and the bottom image shows the same disk upon $980 \mathrm{~nm}$ laser illumination. A computer-controlled laser scanning assembly was used to generate the three dimensional image shown. Reproduced with permission from [33], F. Wang et al., Nature 463, 1061 (2010). (C) 2010, Nature Publishing Group. variety of substrates. Furthermore, multi-color thin film layers that emit different visible colors upon excitation with the same IR wavelength allow for complex security patterns. ${ }^{74} \mathrm{Kim}$ et al. developed photopatternable security marks using $\mathrm{NaYF}_{4}$ doped with $\mathrm{Yb} / \mathrm{Er}$ or $\mathrm{Yb} / \mathrm{Tm} .{ }^{74}$ Ligand exchange was utilized to replace oleic acid with a photopatternable ligand, which allowed the UCNCs to be dispersed in chloroform and then cured with UV radiation once printed. A schematic of the photopatterning technique is shown in Figure 8.

\subsection{Biological Labeling}

Upconversion literature has seen a recent explosion in the number of biological applications. UCNCs are well suited for biological labeling because they are chemically stable, have low toxicity, ${ }^{75}$ and show intense luminescence when excited by near-IR radiation, which can penetrate tissue much better than higher energy radiation such as UV (as is required with quantum dot labels). In addition, very few biological species fluoresce upon IR excitation, 


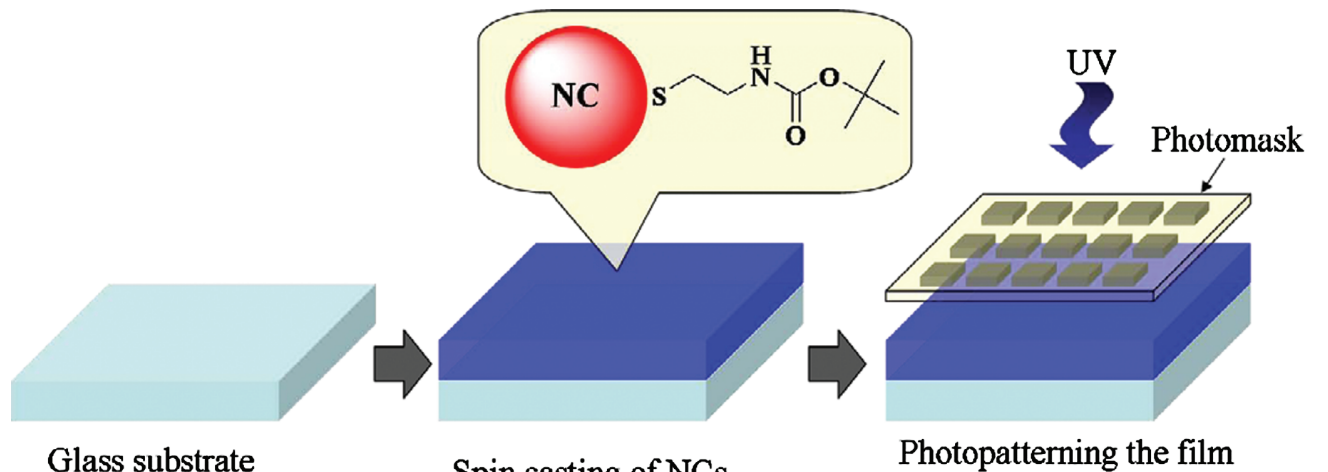

Glass substrate

Spin casting of NCs

through a mask
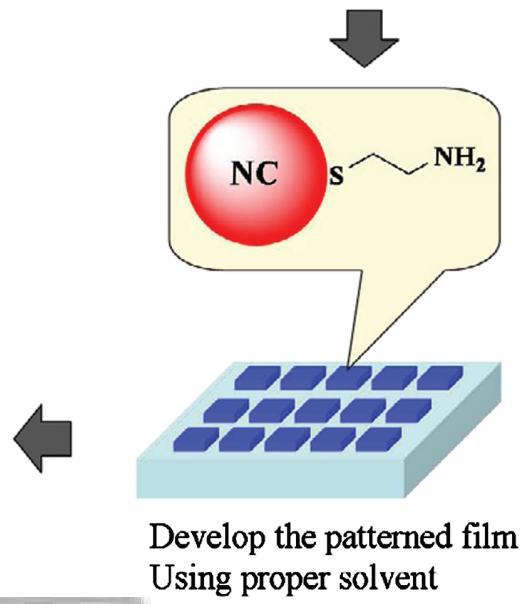

Fig. 8. Schematic diagram of the microlithography photopatterning process used in Ref. [74]. UCNCs with photochromic ligands were first spun onto a glass substrate. UV light was then shone through an optical mask to expose desired areas, and the resulting pattern was developed using hexane. Reproduced with permission from [74], W. J. Kim et al., Nanotechnology 20, 7 (2009). (c) 2009, IOP Publishing.

enabling UCNCs to emit clear signals with very little background "noise" from surrounding molecules. The sharp, well-resolved emission peaks of UCNCs allows them to be easily identified as labels, and multicolor emission upon a single excitation wavelength allows for multiplexing. The ability to make small $(<10 \mathrm{~nm})$, hyrdophilic crystals that can be functionalized with biological molecules has enabled many in vitro and in vivo studies.

Van Veggel's group has recently used PEG-phosphatecoated $\mathrm{NaYF}_{4}$ UCNCs to image ovarian cancer cells in vitro as shown in Figure 9..$^{55}$ Adding an inert shell of $\mathrm{NaYF}_{4}$ to the active core before coating with polymer ligands was shown to increase emission intensity 15 times compared to the core alone. Zhang's group was the first to report in vivo imaging of small mammals using UCNCs. ${ }^{76}$ Polyethyleneimine (PEI) was coated onto $\mathrm{NaYF}_{4}$ nanoparticles, which were subsequently injected into Wistar rats. After a deep tissue injection of $100 \mu \mathrm{L}$ of solution containing $\mathrm{PEI} / \mathrm{NaYF}_{4}: \mathrm{Yb}, \mathrm{Er}$ particles, fluorescence was visible from tissue depths down to $10 \mathrm{~mm}$. Further work by Zhang has shown that live transplanted cells can be tracked in vitro and in vivo using silica/ $\mathrm{NaYF}_{4}: \mathrm{Yb}, \mathrm{Er}$ UCNCs. ${ }^{77}$ Good biocompatibility was found in rats that were tested;
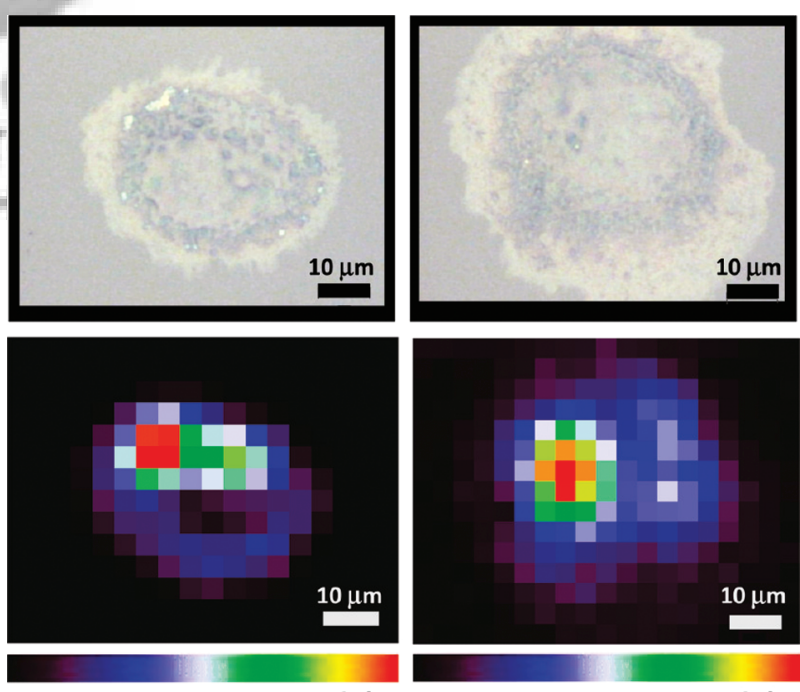

High

Low

High

Fig. 9. In vitro imaging of ovarian cancer cells using PEG-phosphate coated $\mathrm{NaYF}_{4}: \mathrm{Yb}, \mathrm{Tm}$ UCNCs (bottom). A $980 \mathrm{~nm}$ laser diode was used to excite the nanoparticles and confocal microscopy was used to collect the $800 \mathrm{~nm}$ emission given off by the UCNCs. Bright field images of the cells are shown above for comparison. Reproduced with permission from [55], J. C. Boyer et al., Langmuir 26, 1157 (2010). (C) 2010, American Chemical Society. 

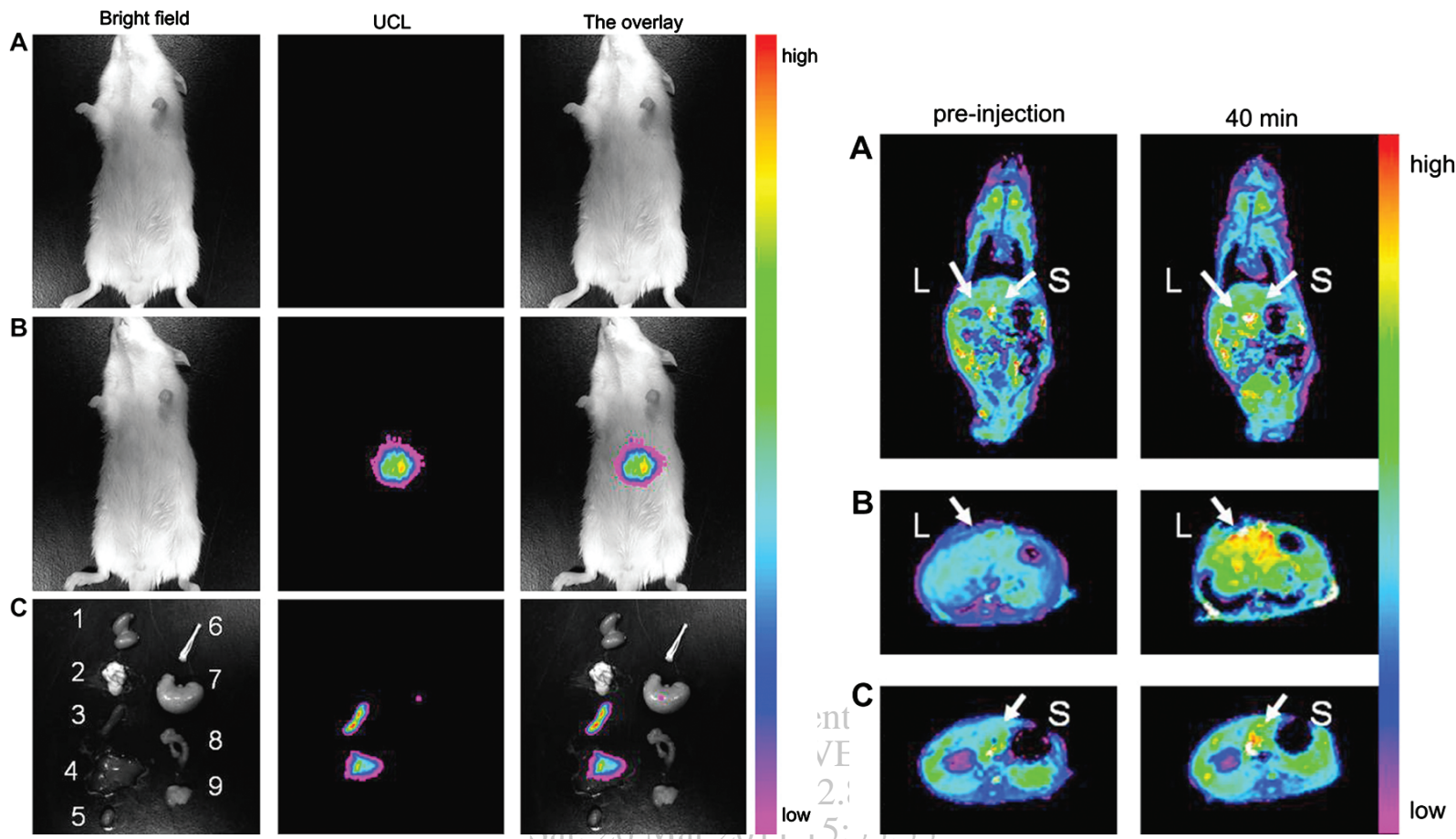

Fig. 10. Upconversion (left) and color-mapped coronal MRI (right) imaging of a mouse using dual-modal UCNCs. Upconversion images show the distribution of UCNCs in vivo (A and B) and ex vivo (C) 40 min after intravenous injection into the mouse's tail. The right side of the figures shows MRI images of the entire mouse (A), a cross-section of the liver (L) and spleen (S) before injection (B), and the liver and spleen 40 min after injection (C). Reproduced with permission from [78], J. Zhou et al., Biomaterials 31, 3287 (2010). (C) 2010, Elsevier.

high resolution, real-time images of transplanted cells were available up to one week after implantation.

Dual-modal UCNCs have also demonstrated great potential for medical imaging purposes. Magnetic resonant imaging (MRI) has been used for many years and is known to give high 3D spatial resolution, but it suffers from low screening sensitivity. ${ }^{66}$ Fluorescent materials have high detection sensitivity but low 3D resolution. Recently, reports have emerged of hybrid paramagnetic/upconverting nanocrystals with the complementary properties of optical and magnetic probes. Li et al. have developed core/shell nanoparticles comprised of $\mathrm{NaYF}_{4}: \mathrm{Yb}$,Er cores with SiDTTA-Gd shells, where Si-DTTA-Gd is an MRI contrast agent. ${ }^{66}$ Zhou et al. have demonstrated the use of dual-modal nanocrystals to image Kunming mice in vivo ${ }^{78}$ Near-infrared to near-infrared UCNCs were used to increase the possible imaging depth and $\mathrm{NaGdF}_{4}$ was chosen as the host material for its paramagnetic relaxivity. Ligand oxidation was utilized to replace oleic acid with azelaic acid ligands on the surface of $\mathrm{NaGdF}_{4}: \mathrm{Yb}, \mathrm{Er}, \mathrm{Tm}$ UCNCs, allowing for water solubility. Figure 10 shows the luminescence and MRI images acquired after the injection of $100 \mu \mathrm{L}$ of nanoparticle solution into the tails of mice. Multifunctional UCNCs such as those described above have the potential to serve as versatile imaging probes for both preoperative diagnosis and post-treatment drug and cell tracking.

\section{CONCLUSIONS AND OUTLOOK}

In summary, we provided a broad overview of the synthesis, properties, assembly and applications of lanthanide doped UCNCs. The recent development of water dispersible, core/shell structures has led to a flurry of research activities primarily geared towards biomedical endeavors. As the field of upconversion nanocrystals continues to evolve from fundamental research to real-world applications, the extent of UCNC utility will only be realized through collaborative efforts among many disciplines. For example, input from the medical community will be necessary to allow widespread use of UCNCs for biological imaging. Furthermore, many areas of research are wide open for discovery. The self-assembly of UCNCs into novel photonic structures and the use of plasmonic coupling with upconversion fluorescence are budding areas of study, promising a myriad of intriguing uses yet to be realized. Significant challenges remain for UCNCs to be more widely applied, such as improved efficiency from smaller particles. However, the future of upconversion benefits from a strong and thriving research base, which sets the stage for both fundamental and applied advancements in the years to come.

\section{References and Notes}

1. F. Auzel, Chem. Rev. 104, 139 (2004).

2. R. Scheps, Prog. Quantum Electron. 20, 271 (1996). 
3. M. R. Brown and W. A. Shand, Phys. Rev. Lett. 12, 367 (1964).

4. L. Esterowi, A. Schnitzl, J. Noonan, and J. Bahler, Appl. Optics 7, 2053 (1968).

5. $\overline{\text { E. Downing, L. Hesselink, J. Ralston, and R. Macfarlane, Science }}$ 273, 1185 (1996).

6. B. M. van der Ende, L. Aarts, and A. Meijerink, Phys. Chem. Chem. Phys. 11, 11081 (2009).

7. J. García Solé, An Introduction to the Optical Spectroscopy of Inorganic Solids, edited by L. E. Bausá and D. Jaque, J. Wiley, Chichester, England, Hoboken, NJ (2005).

8. G. S. Yi, H. C. Lu, S. Y. Zhao, G. Yue, W. J. Yang, D. P. Chen, and L. H. Guo, Nano Lett. 4, 2191 (2004).

9. S. Heer, K. Kompe, H. U. Gudel, and M. Haase, Adv. Mater. 16, 2102 (2004).

10. Z. Q. Li and Y. Zhang, Nanotechnology 19, 345606 (2008).

11. Z. Q. Li and Y. Zhang, Angew. Chem. Int. Ed. 45, 7732 (2006).

12. J. C. Boyer, L. A. Cuccia, and J. A. Capobianco, Nano Lett. 7,847 (2007).

13. F. Wang and X. G. Liu, Chem. Soc. Rev. 38, 976 (2009).

14. E. M. Chan, C. X. Xu, A. W. Mao, G. Han, J. S. Owen, B. E. Cohen, and D. J. Milliron, Nano Lett. 10, 1874 (2010).

15. L. F. Liang, H. F. Xu, Q. Su, H. Konishi, Y. B. Jiang, M. M. Wu, Y. F. Wang, and D. Y. Xia, Inorg. Chem. 43, 1594 (2004)

16. J. H. Zeng, J. Su, Z. H. Li, R. X. Yan, and Y. D, Li, Adv Mater. 17, 2119 (2005).

17. A. Patra, C. S. Friend, R. Kapoor, and P. N. Prasad, Chem: Mater: 15, 3650 (2003)

18. E. De la Rosa-Cruz, L. A. Diaz-Torres, R. A. Rodriguez-Rojas, M. A. Meneses-Nava, O. Barbosa-Garcia, and P. Salas, Appl. Phys. Lett. 83, 4903 (2003)

19. H. Guo, N. Dong, M. Yin, W. P. Zhang, L. R. Lou, and S. D. Xia, J. Phys. Chem. B 108, 19205 (2004).

20. M. Daldosso, D. Falcomer, A. Speghini, M. Bettinelli, S. Enzo, B. Lasio, and S. Polizzi, J. Alloy. Compd. 451, 553 (2008)

21. J. P. Yang, Y. H. Deng, Q. L. Wu, J. Zhou, H. F. Bao, Q. Li, F. Zhang, F. Y. Li, B. Tu, and D. Y. Zhao, Langmuir 26, 8850 (2010).

22. F. Vetrone, J. C. Boyer, J. A. Capobianco, A. Speghini, and M. Bettinelli, J. Phys. Chem. B 107, 10747 (2003).

23. X. Qin, T. Yokomori, and Y. G. Ju, Appl. Phys. Lett. 90, 3 (2007).

24. W. J. Kong, J. Shan, and Y. G. Ju, Mater. Lett. 64, 688 (2010).

25. H. X. Mai, Y. W. Zhang, L. D. Sun, and C. H. Yan, J. Phys. Chem. C 111, 13721 (2007).

26. G. K. Liu, H. Z. Zhuang, and X. Y. Chen, Nano Lett. 2, 535 (2002)

27. J. N. Shan and Y. G. Ju, Nanotechnology 20, 13 (2009).

28. A. Patra, P. Ghosh, P. S. Chowdhury, M. Alencar, B. W. Lozano, N. Rakov, and G. S. Maciel, J. Phys. Chem. B 109, 10142 (2005).

29. F. Vetrone, J. C. Boyer, J. A. Capobianco, A. Speghini, and M. Bettinelli, J. Appl. Phys. 96, 661 (2004).

30. G. Y. Chen, Y. G. Zhang, G. Somesfalean, Z. G. Zhang, Q. Sun, and F. P. Wang, Appl. Phys. Lett. 89, 3 (2006).

31. F. Wang and X. G. Liu. J. Am. Chem. Soc. 130, 5642 (2008).

32. G. F. Wang, Q. Peng, and Y. D. Li, Chem. Eur. J. 16, 4923 (2010).

33. F. Wang, Y. Han, C. S. Lim, Y. H. Lu, J. Wang, J. Xu, H. Y. Chen, C. Zhang, M. H. Hong, and X. G. Liu, Nature 463, 1061 (2010).

34. V. K. Komarala, Y. J. Wang, and M. Xiao, Chem. Phys. Lett. 490, 189 (2010).

35. L. Y. Wang and Y. D. Li, Nano Lett. 6, 1645 (2006).

36. K. W. Kramer, D. Biner, G. Frei, H. U. Gudel, M. P. Hehlen, and S. R. Luthi, Chem. Mater. 16, 1244 (2004).

37. S. Schietinger, L. D. Menezes, B. Lauritzen, and O. Benson, Nano Lett. 9, 2477 (2009).

38. J. N. Shan, M. Uddi, R. Wei, N. Yao, and Y. G. Ju, J. Phys. Chem. C 114, 2452 (2010).

39. D. K. Ma, D. P. Yang, J. L. Jiang, P. Cai, and S. M. Huang, Crystengcomm. 12, 1650 (2010).

40. M. Pollnau, D. R. Gamelin, S. R. Luthi, H. U. Gudel, and M. P. Hehlen, Phys. Rev. B 61, 3337 (2000).
41. J. F. Suyver, A. Aebischer, D. Biner, P. Gerner, J. Grimm, S. Heer, K. W. Kramer, C. Reinhard, and H. U. Gudel, Opt. Mater. 27, 1111 (2005).

42. J. F. Suyver, A. Aebischer, S. Garcia-Revilla, P. Gerner, and H. U. Gudel, Phys. Rev. B 71, 9 (2005).

43. J. W. Zhao, Y. J. Sun, X. G. Kong, L. J. Tian, Y. Wang, L. P. Tu, J. L. Zhao, and H. Zhang, J. Phys. Chem. B 112, 15666 (2008).

44. H. Guo, Z. Q. Li, H. S. Qian, Y. Hu, and I. N. Muhammad, Nanotechnology 21, 6 (2010).

45. L. P. Qian, D. Yuan, G. S. Yi, and G. M. Chow, J. Mater. Res. 24, 3559 (2009).

46. G. S. Yi and G. M. Chow, Chem. Mater. 19, 341 (2007).

47. J. Pichaandi, F. van Veggel, and M. Raudsepp, ACS Appl. Mater. \& Interface 2, 157 (2010).

48. F. Vetrone, R. Naccache, V. Mahalingam, C. G. Morgan, and J. A. Capobianco, Adv. Funct. Mater. 19, 2924 (2009).

49. N. J. J. Johnson, N. M. Sangeetha, J. C. Boyer, and F. van Veggel, Nanoscale 2, 771 (2010).

50. Z. Y. Ma, D. Dosev, M. Nichkova, S. J. Gee, B. D. Hammock, and I. M. Kennedy, J. Mater. Chem. 19, 4695 (2009).

51. Z. Q. Li, Y. Zhang, and S. Jiang, Adv. Mater. 20, 4765 (2008).

52. D. K. Yi, S. S. Lee, G. C. Papaefthymiou, and J. Y. Ying, Chem. Mater 18, 614 (2006).

53. D. Li, B. A. Dong, X. Bai, Y. Wang, and H. W. Song, J. Phys. Chem. C 114, 8219 (2010).

54. R. De Palma, S. Peeters, M. J. Van Bael, H. Van den Rul, K. Bonroy, W. Laureyn, J. Mullens, G. Borghs, and G. Maes, Chem. Mater. 19, 1821 (2007).

55. J. C. Boyer, M. P. Manseau, J. I. Murray, and F. van Veggel, Langmuir 26, 1157 (2010)

56. R. Kumar, M. Nyk, T. Y. Ohulchanskyy, C. A. Flask, and P. N. Prasad, Adv. Funct. Mater. 19, 853 (2009).

57. Z. G. Chen, H. L. Chen, H. Hu, M. X. Yu, F. Y. Li, Q. Zhang, Z. G. Zhou, T. Yi, and C. H. Huang, J. Am. Chem. Soc. 130, 3023 (2008).

58. H. P. Zhou, C. H. Xu, W. Sun, and C. H. Yan, Adv. Funct. Mater. 19, 3892 (2009).

59. G. Decher, Science 277, 1232 (1997).

60. L. Y, Wang, R. X. Yan, Z. Y. Hao, L. Wang, J. H. Zeng, H. Bao, X. Wang, Q. Peng, and Y. D. Li, Angew. Chem. Int. Ed. 44, 6054 (2005).

61. F. Tam, G. P. Goodrich, B. R. Johnson, and N. J. Halas, Nano Lett. 7, 496 (2007).

62. R. Esteban, M. Laroche, and J. J. Greffet, J. Appl. Phys. 105, 10 (2009).

63. S. Schietinger, T. Aichele, H. Q. Wang, T. Nann, and O. Benson, Nano Lett. 10, 134 (2010).

64. H. Zhang, Y. J. Li, I. A. Ivanov, Y. Q. Qu, Y. Huang, and X. F. Duan, Angew. Chem. Int. Ed. 49, 2865 (2010).

65. F. Zhang, G. B. Braun, Y. F. Shi, Y. C. Zhang, X. H. Sun, N. O. Reich, D. Y. Zhao, and G. Stucky, J. Am. Chem. Soc. 132, 2850 (2010).

66. Z. Q. Li, Y. Zhang, B. Shuter, and N. M. Idris, Langmuir 25, 12015 (2009).

67. F. Zhang, Y. G. Deng, Y. F. Shi, R. Y. Zhang, and D. Y. Zhao, J. Mater. Chem. 20, 3895 (2010).

68. C. Chen, L. D. Sun, Z. X. Li, L. L. Li, J. Zhang, Y. W. Zhang, and C. H. Yan, Langmuir 26, 8797 (2010).

69. A. Shalav, B. S. Richards, and M. A. Green, Sol. Energy Mater. Sol. Cells. 91, 829 (2007).

70. T. Trupke, M. A. Green, and P. Wurfel, J. Appl. Phys. 92, 4117 (2002).

71. C. Zhang, H. P. Zhou, L. Y. Liao, W. Feng, W. Sun, Z. X. Li, C. H. Xu, C. J. Fang, L. D. Sun, Y. W. Zhang and others, Adv. Mater. 22, 633 (2010). 
72. R. T. Chai, H. Z. Lian, Z. Y. Hou, C. M. Zhang, C. Peng, and J. Lin, J. Phys. Chem. C 114, 610 (2010).

73. J. C. Boyer, N. J. J. Johnson, and F. van Veggel, Chem. Mater. 21, 2010 (2009).

74. W. J. Kim, M. Nyk, and P. N. Prasad, Nanotechnology 20, 7 (2009).

75. R. A. Jalil and Y. Zhang, Biomaterials 29, 4122 (2008).
76. D. K. Chatteriee, A. J. Rufalhah, and Y. Zhang, Biomaterials 29, 937 (2008).

77. N. M. Idris, Z. Q. Li, L. Ye, E. K. W. Sim, R. Mahendran, P. C. L. Ho, and Y. Zhang, Biomaterials 30, 5104 (2009).

78. J. Zhou, Y. Sun, X. X. Du, L. Q. Xiong, H. Hu, and F. Y. Li, Biomaterials 31, 3287 (2010).

Received: 6 July 2010. Accepted: 4 August 2010.

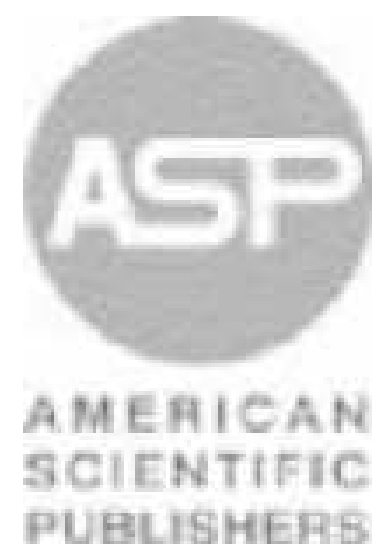

